\title{
Diagnosis and prognosis of malignant mesothelioma of the tunica vaginalis testis
}

\author{
Bo Hai, MD; ${ }^{*}$ Yu Yang, MD; ${ }^{\dagger}$ Yajun Xiao, MD, ${ }^{*}$ Bing Li, MD, ${ }^{*}$ Chaohui Chen, MD*
}

*Department of Urology, Union Hospital, Tongii Medical School, Huazhong University of Science and Technology, Wuhan, China; ${ }^{\dagger}$ Center for Molecular Physiology Research, Children's National Medical Center, Washington DC

Cite as: Can Urol Assoc J 2012;6:E238-E241. http://dx.doi.org/10.5489/cuaj.10200. Epub 2011 Sept 8 .

\section{Abstract}

Objectives: The objective of this study is to evaluate the diagnosis and prognosis of malignant mesothelioma of the tunica vaginalis testis through an additional 6 patients with urogenital mesothelioma.

Methods: Six patients with urogenital mesothelioma who underwent adequate surgical procedures and histopathologic analysis from 1990 to 2009 were identified and retrospectively reviewed.

Results: Six patients between the ages of 26 and 78 years with urogenital mesothelioma, 5 of which originated in the scrotum and 1 in the spermatic cord. Histopathologic analysis showed that CK5/6 and calretinin were positive in all cases, 5 cases were positive for vimentin, and 1 case showed focal weak positive reaction with MOC3, but none of the cases stained for CEA or CD15. The overall recurrence rate of urogenital mesothelioma after surgery was $5 / 6$, including local recurrences and fatalities due to tumour. Conclusions: In cases of mesothelioma of the tunica vaginalis testis, the histopathologic markers we chose helped confirm the histopathological diagnosis; adequate surgical procedures are typically not curative, and this tumour is often fatal.

\section{Introduction}

Malignant mesothelioma is most commonly found in the pleura, peritoneum and pericardium, while mesothelioma involving the tunica vaginalis testis is very rare. ${ }^{1}$ Malignant genital mesothelioma usually presents as a benign hydrocele with a painless lump in the scrotum. A history of asbestos exposure has been reported in $35 \%$ of patients with this disease. ${ }^{2}$ In general, three main histological types of malignant mesothelioma have been described: the epithelial type, the sarcomatous type and the biphasic type. ${ }^{3}$ Plas and colleagues described clinical features and prognostic parameters of mesothelioma of the tunica vaginalis testis, ${ }^{2}$ but there have been few reports of this tumour with an evaluation of immunohistochemical characteristics. This paper describes the clinical features, immunohistochemical findings and prognosis in 6 cases from China; the paper also provides a review of the literature.

\section{Methods}

Six samples, originally identified as malignant mesothelioma of the tunica vaginalis testis, were selected from the archives of the Department of Pathology (1990 to 2009) at Union Hospital, an associated hospital of Tongji Medical College. Clinical findings included chest X-rays, computed tomography $(\mathrm{CT})$, echogram and routine clinical chemistry and follow-up data for all patients. All 6 patients had a clear clinicopathologic diagnosis of malignant mesothelioma and adequate surgical treatment. The ages of these patients ranged from 26 to 78 years. All patient histories were investigated for asbestos exposure.

Histologic specimens obtained at surgery were fixed in $10 \%$ formalin, routinely processed and embedded in paraffin All specimens were evaluated immunohistochemically with antibodies calretinin, vimentin, CK5/6, MOC31, CD15 and CEA using staining procedures. Sections were deparafinzed and rehydrated, and endogenous peroxidase was blocked by treatment with $0.3 \%$ hydrogen peroxide in methanol for 30 minutes. Sections were incubated with normal horse serum for 20 minutes and incubated overnight at $4{ }^{\circ} \mathrm{C}$ with mouse-antihuman monoclonal antibodies (Santa Cruz Biotechnology, Santa Cruz, CA). Reactions to identify positive antibody reactions were carried out with a commercial streptavidin-biotin peroxidase labelling kit (Boster Corp., China).

\section{Results}

Clinical findings are summarized in Table 1. In four cases (cases 2,4,5,6), the tumour affected the scrotum (cases 2, 4, 5 and 6); in two cases, it affected the epididymis (Fig. 1) and the spermatic cord (cases 1 and 3). Two patients (cases 2 and 


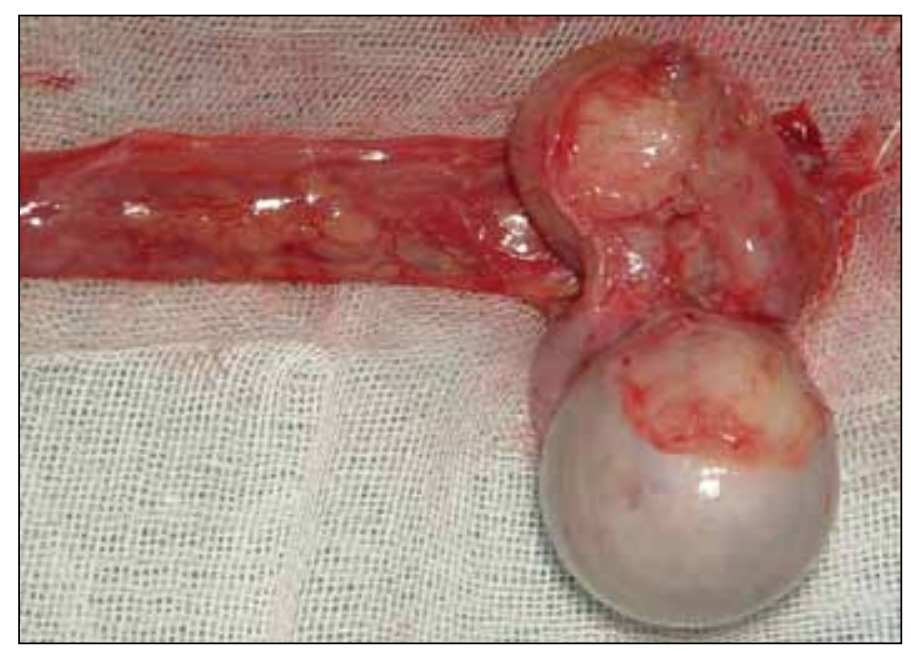

Fig. 1. Gross photograph of radical orchiectomy specimen. Pale and friable lesions are visible on the tunica albuginea and epididymis, invading the testicular parenchyma surface.

5) had long-standing bilateral hydrocele, and five patients complained of painless enlargement of the scrotum or the spermatic cord. One patient (case 4) presented with pain of the appendix testis pain which went on for several months. Physical examination revealed a suspicious inguinal lymph node in one case (case 5).

Ultrasonography demonstrated an irregular thickening of the tunica vaginalis testis in all cases and increased blood flow to the epididymis testis. A CT scan of the chest, abdomen and pelvis was negative for metastatic disease in three patients; another three patients presented with findings suspicious for retroperitoneal lymph node involvement (cases 4 and 6) and pulmonary (3 and 6) metastasis. Two patients (4 and 6) had a history suggestive of exposure to asbestos. Radical scrotal orchiectomy with or without an inguinal

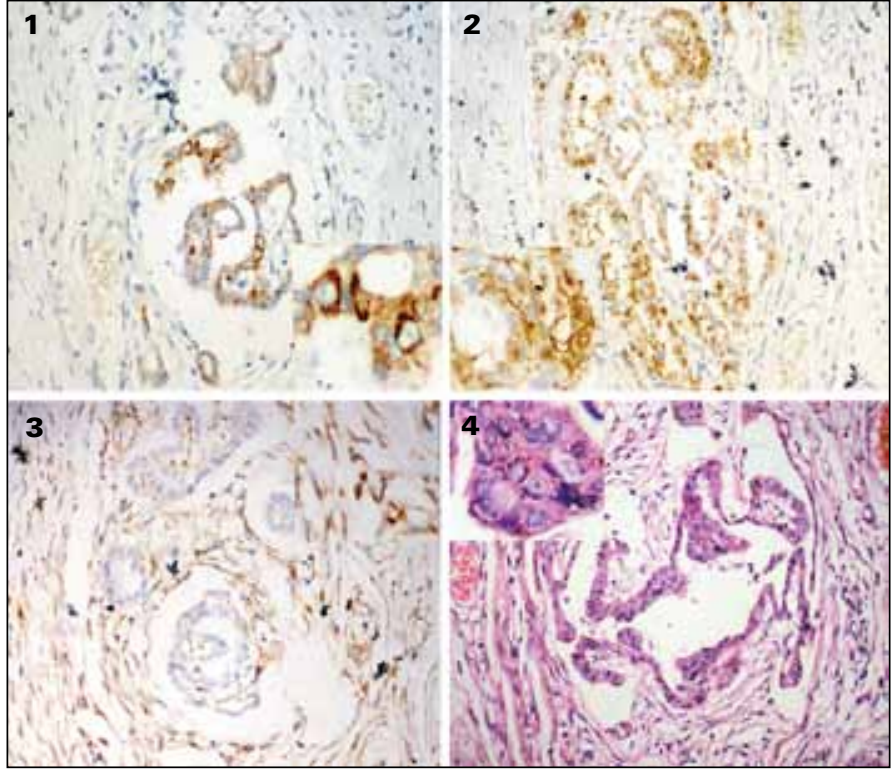

Fig. 2. Immunohistochemistry of malignant mesothelioma of the tunica vaginalis testis: 1. Malignant mesothelioma positive for $\mathrm{CK} 5 / 6$ with perinuclear enhancement (case 3); 2. Malignant mesothelioma showing nuclear and cytoplasmic positivity for Calretinin (case 3); 3 . Malignant mesothelioma positive for Vimentin in rhabdoid cells (case 6); 4. Malignant epithelial mesothelioma exhibiting a papillary growth pattern with nesting of cuboidal tumour cells showing acidophilic cytoplasm and vesicular nuclei. $H \& E$ stain. (case 3). Magnification at $\times 400$.

lymph node dissection was carried out in four cases (cases 2, 4, 5 and 6), and two patients were treated with a local tumour resection (cases 1 and 3). Adjuvant therapy was carried out in four cases (combined chemotherapy and radiotherapy in one case, chemotherapy only in one case, and radiotherapy in two cases; systemic chemotherapy was with doxorubicin). In the 2-year follow-up, two patients died due to the tumour, three patients experienced local tumour recurrence, and only

Table 1. Pretreatment characteristics, treatment and outcome in 6 patients with malignant mesothelioma of the tunica vaginalis: chemotherapy with doxorubicin

\begin{tabular}{|c|c|c|c|c|c|c|c|}
\hline Case & Age & $\begin{array}{l}\text { Asbestos } \\
\text { exposure }\end{array}$ & Physical findings & $\begin{array}{c}\text { Pretreatment } \\
\text { suspicion for } \\
\text { metasasis }\end{array}$ & Surgery & Adjuvant therapy & 2 Years Follow-up \\
\hline 1 & 26 & No & $\begin{array}{l}\text { Left spermatic cord } \\
\text { mass }\end{array}$ & No & LTR; ILND & Radiotherapy & No recurrence \\
\hline 2 & 67 & No & $\begin{array}{l}\text { Left scrotal mass; } \\
\text { Bilateral hydrocele }\end{array}$ & No & RSO; ILND & Chemotherapy & Local recurrence \\
\hline 3 & 57 & No & $\begin{array}{l}\text { Right spermatic } \\
\text { cord mass }\end{array}$ & Lung & LTR & $\begin{array}{c}\text { Chemotherapy ; } \\
\text { Radiotherapy }\end{array}$ & Local recurrence \\
\hline 4 & 46 & Yes & $\begin{array}{l}\text { Left scrotal mass; } \\
\text { Acute appendix } \\
\text { testis pain; }\end{array}$ & $\begin{array}{l}\text { Retroperitoneal } \\
\text { lymph nodes }\end{array}$ & RSO & Radiotherapy & Dead of disease \\
\hline 5 & 78 & No & $\begin{array}{l}\text { Left scrotal mass; } \\
\text { Bilateral hydrocele }\end{array}$ & $\begin{array}{l}\text { Inguinal lymph } \\
\text { node }\end{array}$ & RSO & None & Local recurrence \\
\hline 6 & 76 & Yes & Right scrotal mass & $\begin{array}{l}\text { Retroperitoneal } \\
\text { lymph nodes; Lung }\end{array}$ & RSO & None & Dead of disease \\
\hline
\end{tabular}


Hai et al.

\begin{tabular}{lcccccc}
\hline \multicolumn{7}{c}{ Table 2. Antibodies reactivity in 6 patients } \\
\cline { 2 - 7 } Case & Calretinin & CK5/6 & Vimentin & MOC 31 & $\begin{array}{c}\text { CD15 } \\
\text { (LeuM1) }\end{array}$ & CEA \\
\cline { 2 - 7 } & Cimody \\
\hline 1 & + & + & + & - & - & - \\
2 & + & + & + & - & - & - \\
3 & + & + & - & - & - & - \\
4 & + & + & + & - & - & - \\
5 & + & + & + & $+/-$ & - & - \\
6 & + & + & + & - & - & - \\
\hline
\end{tabular}

+: positive; -: negative; +/-: focal weak positive.

one patient showed no evidence of disease.

All cases were predominantly epithelial type mesothelioma with a diffuse pattern of proliferation (Fig. 1). Immunohistochemical staining showed CK5/6 and calretinin positivity in all cases (Fig. 2), and five cases were positive for vimentin (Fig 2). All cases were negative for CEA and CD15 (Fig. 2). One case showed a weak positive reaction to MOC31 (case 2) (Fig. 2). This pattern of immunostaining with clinicohistopathologic findings was diagnostic of malignant mesothelioma (Table 2).

\section{Discussion}

Mesothelioma is an uncommon malignancy arising from the epithelial membranes of the coelomic cavities, such as the pleura, pericardium and peritoneum. ${ }^{4}$ Malignant mesothelioma originating from the tunica vaginalis is extremely rare. About 100 cases have been reported since its first description by Barbera and Rubino in $1957 .{ }^{5}$ The tumour occurs most frequently in older men; 5 of our cases were patients over 45 , consistent with previous findings. ${ }^{2}$ Two cases (cases 4 and 6) had a history suspicious for asbestos exposure, and two cases (cases 2 and 5) had scrotal hydrocele. Although the exact pathophysiology is unclear, contact with asbestos and long-standing hydrocele are considered to be risk factors of malignant mesothelioma of the tunica vaginalis., ${ }^{2,6,7}$ Radical orchiectomy through an inguinal approach is recommended with hemiscrotectomy and local excision of the spermatic cord. In two cases, patients with regional lymph node metastases developed distant metastases and died of neoplasm, and two cases of local recurrence cases developed from involved inguinal nodes. Inguinal lymphadenectomy should, therefore, be considered once the primary diagnosis has been established. However, even after radical orchiectomy and adjuvant therapy, the overall recurrence rate was still high (2 dead of disease, and 3 local recurrences), which is higher than the previous 53\% reported recurrence rate in a large study. ${ }^{2}$ As such, chemotherapy (e.g., doxorubicin) and/ or radiotherapy appear to have limited impact in advanced disease. The common sites of metastasis in our cases were inguinal nodes, retroperitoneal lymph nodes and lung. We believe that adjuvant radiotherapy should be considered in these regions, regardless of whether surgical margins appear free of tumour, or whether patients have disseminated disease. Although radiotherapy may not improve overall survival, it can provide better local control of neoplasm.

The expression of both epithelial and mesenchymal markers is a constant feature in malignant mesothelioma, which may be related to the origin of mesothelial cells. ${ }^{8}$ The histopathologic differential diagnoses of malignant urogenital mesotheliomas includes metastatic adenocarcinoma, papillary mesothelial hyperplasia, carcinoma of the rete testis and embryonal carcinoma. Calretinin expression by mesothelial cells and its positivity in epithelial mesothelioma may be useful in distinguishing it from adenocarcinoma and mesothelial hyperplasia, which are usually calretinin negative. Comin and colleagues concluded that calretinin was the most sensitive $(100 \%)$ and specific $(91.3 \%)$ of mesothelioma markers. ${ }^{9}$ Cytokeratin (CK) intermediate filaments are part of the cytoskeleton of epithelial cells. Twenty different types of CKs may be found in human epithelia. ${ }^{10} \mathrm{CK} 5 / 6$ has high sensitivity and specificity for epithelial mesothelioma and is now recognized as a valuable marker in distinguishing this tumour from adenocarcinoma and sarcoma. ${ }^{11}$ In our study, calretinin and CK5/6 were positive in all cases, confirming the value of coexpression of calretinin and CK5/6 in thediagnosis of malignant mesothelioma. Vimentin is also useful as a positive marker for mesothelioma. Kayser and colleagues reported $83 \%$ of malignant mesotheliomas were positive for vimentin. ${ }^{12}$ In our study, we detected vimentin positivity in 4 of 6 cases, but this antibody may be positive more frequently in cases of biphasic and sarcomatoid mesothelioma. ${ }^{13}$ None of our cases stained for CEA or CD15 (LeuM1), and it has been recommended that these antibodies be used for separating mesothelioma from adenocarcinoma and other epithelial neoplasms, as CD15 and CEA generally lack reactivity with mesothelioma cells. ${ }^{14}$

\section{Conclusion}

We presented 6 rare cases of malignant mesothelioma of the tunica vaginalis testis from China. Consistent with previously reported cases, the prognosis for this tumour remains poor despite aggressive surgical procedures and adjuvant therapies. Because of the high potential for misdiagnosis, a panel of immunohistochemical markers is required for a definitive diagnosis.

Competing interests: None declared.

This paper has been peer-reviewed. 


\section{References}

1. Gupta NP, Agrawal AK, Sood S, et al. Malignant mesothelioma of the tunica vaginalis testis: A report of two cases and review of the literature. J Surg Oncol 1999;70:251-4.

2. Plas E, Riedl CR, Pflüger H. Malignant mesothelioma of the tunica vaginalis testis. Review of the literature and assessment of prognostic parameters. Cancer 1998;83:2437-46.

3. Chen IL, Hsu YH. Malignant mesothelioma of the tunica vaginalis testis: a case report and literature review. Kaohsiung J Med Sci 2009 Feb;25:77-81.

4. Antman K, Hassan R, Eisner M, et al. Update on malignant mesothelioma. Oncology (Williston Park) 2005;19:1301-9; discussion 1309-10, 1313-6.

5. Barbera V, Rubino M. Papillary mesothelioma of tunica vaginalis, Cancer 1957; 10(1):183-9.

6. Jones MA, Young RH, Scully RE. Malignant mesothelioma of the tunica vaginalis. A clinicopathologic analysis of 11 cases with review of the literature. Am J Surg Pathol 1995;19(7):815-25.

7. Gurdal $M$, Erol A. Malignant mesothelioma of tunica vaginalis testis associated with long-lasting hydrocele:could hydrocele be an etiological factor? Int Urol Nephrol 2001;32:687-9.

8. Mutsaers S.E.The mesothelial cell. Int J Biochem Cell Biol 200436:9-16.

9. Comin CE, Luca N, Vieri B, et al.Calretinin, Thrombomodulin, CEA, and CD15: A useful combination of immunohistochemical markers for differentiating pleural epithelioid mesothelioma from peripheral pulmonary adenocarcinoma. Hum Pathol 2001;32:529-36.
10. Cooper D, Schermer A, Sun TT. Classification of human epithelia and their neoplasms using monoclonal antibodies to keratins: Strategies, applications and limitations. Lab Invest 1985;52:243-56.

11. Ordonez, N. G. Immunohistochemical diagnosis of epithelioid mesothelioma: a critical review of old markers, new markers. Human Pathology 2002;33:953-967.

12. Kayser $K$, Bohm G, Blum S, et al.Glyco-and immunohistochemical refinement of the differential diagnosis between mesothelioma and metastatic carcinoma and survival analysis of patients. J Pathol 2001;193: 175-80.

13. Mayall FG, Goddard H, Gibbs AR. Intermediate filament expression in mesotheliomas: Leimyoid mesotheliomas are not uncommon. Histopathology 1992;21:453-7.

14. Comin CE, Luca N, Vieri B, Paqlierani M, Dini S.Calretinin, Thrombomodulin, CEA, and CD15: A useful combination of immunohistochemical markers for differentiating pleural epithelioid mesothelioma from peripheral pulmonary adenocarcinoma. Hum Pathol 2001;32:529-36.

Correspondence: Dr. Chaohui Chen, Department of Urology, Union Hospital, Union Hospital, Wuhan, China, 43002; chenchaohui02@126.com 